\title{
Introduction to Securing Knowledge Systems and Managing Knowledge Risks Minitrack
}

\author{
Alexandra Durcikova \\ University of Oklahoma \\ alex@ou.edu
}

\author{
Murray E. Jennex \\ San Diego State University \\ mjennex@mail.sdsu.edu
}

\author{
Ilona Ilvonen \\ Tampere University \\ olona.ilvonen@tuni.fi
}

The purpose of this Minitrack is to focus on research on the intersection of knowledge systems, knowledge management, security, and risk management. It seeks papers that investigate issues related to security and protection of intellectual assets and explore how organizations can use security measures to protect their KM practices.

During the nine years of existence of this minitrack, we have published twenty-one papers that focus on the intersection of knowledge management and organizational or individual security and knowledge risk. These papers belong to one of the following emerging themes: (1) Protecting Confidentiality of Knowledge; (2) Protecting Integrity of Knowledge; (3) Protecting Knowledge Loss Risk; and (4) Improving Knowledge of Safe Cyber Behavior.

This year's papers follow the tradition of bringing papers that are at the intersection of security and KM; both spam across multiple themes. The first paper by AJ Burns discusses how the regulatory focus on rational choices influences employees' intention to protect organizational information assets. The result of these exploratory analyses indicates that promotion and prevention foci each distinctly relate to perceived costs and benefits of protecting organizational information assets. Additionally, the findings show that the overall benefit of protecting mediates an expanded set of costs and benefits

The second paper, by Fruhwirth, PammerShindler, and Thalmann, explores data-driven business models and ways they create unwanted knowledge spillovers. The authors developed a network-based representation of data-driven business models within one case organization and identified knowledge risks using 17 interviews with experts from the domain of business models, data analysis, and knowledge management. We found that the network-based representation is suitable to visualize, discuss, and create awareness for knowledge risks and see types of data-related value objects and quantification of risks as two recommendations for further research.

The minitrack co-chairs want to thank authors and reviewers for their work in making this nine-year of the minitrack a success. We encourage authors whose research focus is on the intersection of knowledge management and individual or organizational security to submit their work to this minitrack in the future. Research focusing on cybersecurity training is also welcome. 